\title{
Direct Observation of the Dynamics of Self-Assembly of Individual Solvation Layers in Molecularly Confined Liquids
}

\author{
Josep Relat-Goberna and Sergi Garcia-Manyes* \\ Department of Physics and Randall Division of Cell and Molecular Biophysics, \\ King's College London, Strand, WC2R 2LS London, United Kingdom
}

(Received 11 March 2015; published 25 June 2015)

\begin{abstract}
Confined liquids organize in solidlike layers at the liquid-substrate interface. Here we use force-clamp spectroscopy AFM to capture the equilibrium dynamics between the broken and reformed states of an individual solvation layer in real time. Kinetic measurements demonstrate that the rupture of each individual solvation layer in structured liquids is driven by the rupture of a single interaction for 1-undecanol and by two interactions in the case of the ionic liquid ethylammonium nitrate. Our results provide a first description of the energy landscape governing the molecular motions that drive the packing and self-assembly of each individual liquid layer.
\end{abstract}

PACS numbers: 82.37.Gk, 68.08.De, 68.37.Ps, 82.65.+r

Understanding the structural properties of liquids at the solid interface is fundamental to many applications in the fields of nanotribology, wetting, or molecular biophysics [1]. When confined between two flat surfaces separated by a few nanometers, liquids exhibit properties that cannot be described by the continuum theories based on van der Waals and electrostatic interactions characterizing the bulk properties. Instead, the molecules forming the liquid order into well-defined solvation layers, giving rise to oscillatory forces described by radial distribution functions with a periodicity of about one molecular diameter [1].

The discrete oscillation forces were first experimentally measured by pioneering surface force apparatus (SFA) experiments, in which liquids are confined between two opposing macroscopic flat mica plates of $R \sim 10 \mu \mathrm{m}$ [2]. These observations were later complemented by atomic force microscopy (AFM) measurements, where the confined liquid is compressed by the AFM tip, with a much smaller contact radius $(R \sim 10 \mathrm{~nm})$ [3]. In the latter case, the deflection of the AFM cantilever is measured as it approaches a rigid, flat substrate [typically highly oriented pyrolytic graphite (HOPG) or mica] at constant velocity. The layered nature of the probed liquids is hallmarked by the presence of discrete jumps in the resulting forcedistance curves. Each discontinuity or jump occurs when an individual layer of confined liquid is suddenly squeezed out from below the AFM tip [4]. Crucially, the distance between two consecutive jumps informs on the precise orientation of each molecular layer with respect to the surface. This experimental approach has revealed

Published by the American Physical Society under the terms of the Creative Commons Attribution 3.0 License. Further distribution of this work must maintain attribution to the author(s) and the published article's title, journal citation, and DOI. invaluable information about the molecular packing properties of a variety of chemically distinct liquids, encompassing nonpolar liquids with spherical structure such as octamethylcyclotetrasiloxane (OMCTS) [5], linear and branched alkanes [6,7], polar liquids such as short alcohols [8], a variety of ionic liquids $[9,10]$, and even water [11,12], all exhibiting a priori similar force-induced rupture mechanisms. This progress notwithstanding, the reverse process encompassing the reversible reformation of each individual molecular layer, together with the quantification of the number of interactions involved in the rupture and reformation processes, remained completely elusive.

In this Letter, we make use of force-clamp spectroscopy AFM to directly capture the forced-induced rupture and reformation of each of the individual solidlike ordered layers populating the alcohol-HOPG and ethylammonium nitrate (EAN)-mica interface. These measurements enable us to map out the complete energy landscape of a confined liquid, providing (sub)molecular insight into the mechanisms underpinning the rupture and reformation dynamics of packing and self-assembly of an individual liquid layer according to its particular chemical structure.

Using a homemade AFM spectrometer [13], we measured individual force distance curves [Fig. 1(a)] on 1-undecanol, whereby the cantilever was approached towards (red trace) and, subsequently, retracted from (blue trace) a HOPG surface at a constant velocity of $200 \mathrm{~nm} \mathrm{~s}^{-1}$. Upon approaching the surface, the cantilever penetrated 5 welldefined 1-undecanol layers (red arrows), each of them requiring an exponentially higher force to be indented [Fig. 1(b)]. As the cantilever was withdrawn from the sample, each previously broken layer was reversibly reformed (blue arrows). The distribution of breakthrough forces can be fitted with an exponentially decaying function [4],

$$
F=F_{0} \cos (2 \pi d) \exp (-\tau d)
$$



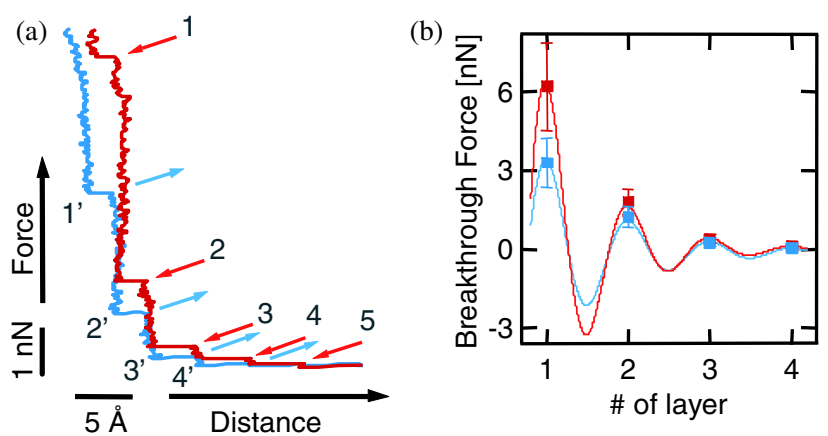

FIG. 1 (color online). Solvation layers in 1-undecanol revealed by constant velocity AFM. (a) Force vs distance plot of the AFM tip approaching towards (red line) and, subsequently, retracting from (blue line) the HOPG surface in 1-undecanol at constant velocity, showing the rupture and reversible reformation of a total of 5 confined layers. (b) Rupturing and reforming forces $(n=90$ individual trajectories) for the first four measurable solvation layers.

(where $d$ is the thickness of the confined layers and $\tau$ stands for the decay length), further confirming the periodic packing properties of 1-undecanol. The average jump-in distance $(\sim 3 \AA)$ is significantly shorter than the 1-undecanol length $(\sim 14.7 \AA)$ [14], thus suggesting an almost flat layering of the 1-undecanol layers on the HOPG surface. These results are in full agreement with previous X-ray [15], SFA [14], force [8], and imaging [16] AFM studies. The direct observation of the discrete reformation events in the force-extension traces [Fig. 1(a), blue arrows] suggests that the rupturing process is reversible and that, by holding the applied force constant throughout the experiment at an "average" set-point value between the rupturing and reformation force, the equilibrium dynamics between the broken and reformed states should be captured. In fact, applying a constant force of $430 \mathrm{pN}$ with the force-clamp setup displayed bistability [Fig. 2(a)]; the layer 3 hopped between the broken and reformed states in real time, with a concomitant change in length $\Delta l=3.3 \pm 2 \AA$, corresponding to the length of an individual layer (Fig. S2 in the Supplemental Material [18]). Changing the applied force to lower $(410 \mathrm{pN})$ and higher $(450 \mathrm{pN})$ values dramatically altered the equilibrium between the reformed and broken states. The thermodynamics of the reformed $\rightleftarrows$ ruptured transition can be evaluated by measuring the relative time abundance of the reformed state $N_{\text {ref }} / N_{\text {total }}$ for each distinct constant force value according to the Boltzmann distribution:

$$
\frac{N_{\text {ref }}}{N_{\text {total }}}=1-\frac{1}{1+\exp \left(\frac{\Delta G-\Delta l F}{k T}\right)},
$$

where $\Delta G$ is the difference in free energy between the broken and reformed states and $\Delta l$ is the physical length distance separating them. Fitting this equation to the experimental

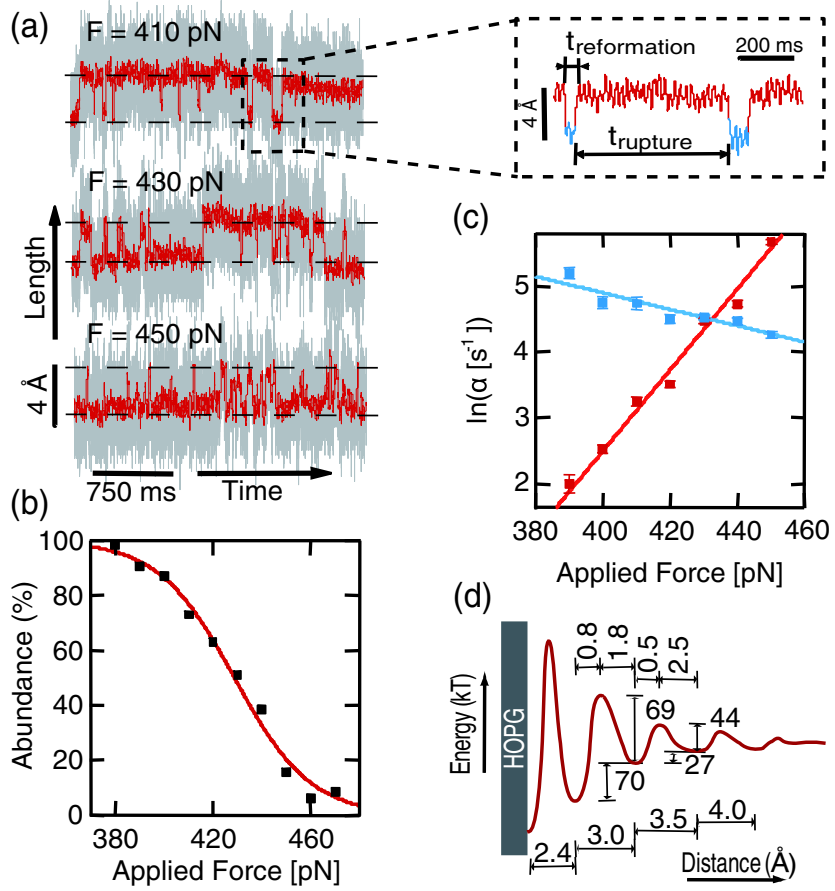

FIG. 2 (color online). The equilibrium dynamics of an individual solvation layer in 1-undecanol captured by force-clamp AFM. (a) Applying a constant force of $430 \mathrm{pN}$ samples the rupture and reformation dynamics of layer 3 . The equilibrium is readily displaced upon changing the applied force. (b) Plot of the abundance of the reformed state as a function of the applied force. (c) The dwell times of rupture and reformation [(a), inset] depend exponentially on the applied force (red and blue dots, respectively). The error has been calculated using the bootstrap method [17]. (d) Repeating the process for each individual layer (Figs. S6-S8) allows the full 1D reconstruction of the energy landscape of confined 1-undecanol.

data corresponding to the rupture and reformation of layer 3 [Fig. 2(b)] yields $\Delta G=(27 \pm 2) k T$ and $\Delta l=2.6 \pm 0.2 \AA$. Notably, the measured value of $\Delta l$ is consistent with the thickness of one individual 1-undecanol layer and in agreement with the results obtained from force-extension (Fig. S1, [18]) and force-clamp (Fig. S2, [18]) measurements. The force dependent rates of breakthrough and reformation have been measured by extracting the dwell time for each individual transition occurring at a fixed force (Fig. S3 [18]), assuming that, as a first approximation, the distribution of dwell times at a particular force follows a single exponential (Fig. S4, [18]). Our observations demonstrate that, both for the rupturing and reformation processes, the measured rates depend exponentially on the applied force following a simple Bell formalism [19]:

$$
\alpha(F)=A_{0} \exp \left(-\frac{\Delta E}{k T}+\frac{\Delta x}{N k T} F\right)
$$

where $\alpha(F)$ is the rate of the process as a function of force, $A_{0}$ the preexponential factor, $\Delta E$ stands for the height of the 
energy barrier, $\Delta x$ is the width of the energy barrier governing the process (normally known as "distance to the transition state") and $N$ is the number of bonds (or interactions) that withstand mechanical force. This approach has been used to interpret a wide variety of single molecule pulling experiments $[13,20,21]$ for which $N=1$. Figure 2(c) shows the graph plotting the $\ln [\alpha(F)]$ as a function of the pushing force, for both the rupturing (red) and reformation (blue) processes. Fitting Eq. (3) for both processes results in $\Delta x_{\text {rupture }} / N=2.5 \pm 0.1 \AA$ for the rupturing process $\Delta x_{\text {reformation }} / N=0.5 \pm 0.1 \AA$ for the reverse reforming reaction. By assuming an arbitrary value $A_{0}=4 \times$ $10^{9} \mathrm{~s}^{-1}$ [22], we obtain $\Delta E=44 k T$.

Dividing the expressions for the rates of rupturing $\alpha_{\text {rup }}$ and reformation $\alpha_{\text {ref }}$ and assuming that $A_{0 \text {,rup }} \sim A_{0 \text {,ref }}$ it follows that

$$
\ln \left(\frac{\alpha_{\text {rup }}}{\alpha_{\text {ref }}}\right)=\frac{\Delta G}{k T}+\frac{\Delta l}{N k T} F .
$$

Fitting Eq. (4) to the experimental data, and using the previously obtained value of $\Delta l=2.6 \pm 0.2 \AA$, we obtain $\Delta G=(31 \pm 2) k T$ and $N=0.9 \pm 0.1$. Remarkably, the $\Delta G$ values obtained through kinetic and thermodynamic measurements are in close agreement, thus strongly suggesting that the hopping process occurs through a single barrier. Most importantly, our experiments revealed that $N \approx 1$ (by definition $N$ must be an integer $\geq 1$ ). This has fundamental implications, entailing that the applied force is transmitted through an "individual" interaction within a very narrow space. These conclusions complement recent findings suggesting that the force does not distribute through the whole cantilever tip in contact with the liquid (which would imply that a larger number of bonds are effectively broken, yielding a large $N$ ) but rather through the last microasperities of the AFM cantilever tip [23]. The same approach used to uncover the kinetic and thermodynamic properties of layer 3 was extended and applied to layers 1 and 2 (Figs. S6-S8 in the Supplemental Material [30]). Combined, these results allowed us to reconstruct the energy landscape of the first confined layers of 1-undecanol in the 1D direction perpendicular to the graphite surface [Fig. 2(d)]. Interestingly, and as expected in light of the large hysteresis between the breakthrough and reformation events observed in Fig. 1(a), the layer closer to the surface does not exhibit signatures of dynamic equilibrium between the ruptured and reformed states. Moreover, in this case, $N$ is an order of magnitude bigger than in the rest of the confined layers (Table SI, [18]). The markedly different behavior of this first layer suggests that the underlying solid substrate is likely to have a large effect on the conformation and dynamics of the layer.

The dynamic properties of a long, nonbranched alcohol, such as 1-undecanol, lying flat on a HOPG surface sets the ground to study the molecular self-assembly mechanism of chemically more complex solvents. Because of their sterically mismatched anion-cation pairs, ionic liquids exhibit fascinating properties that lie in-between those of a liquid and a solid $[9,10]$. In this context, ethylammonium nitrate (EAN) has revealed oscillatory forces in the vicinity of a flat mica substrate. Using constant velocity experiments [Fig. 3(a)], we recorded force-distance traces showing the initial 3 clear discontinuities hallmarking the rupture (red) and reformation (blue) processes. In this case, each jump measures $4.3 \pm 0.4 \AA$ in excellent agreement with the predicted ion pair diameter of EAN and with previous
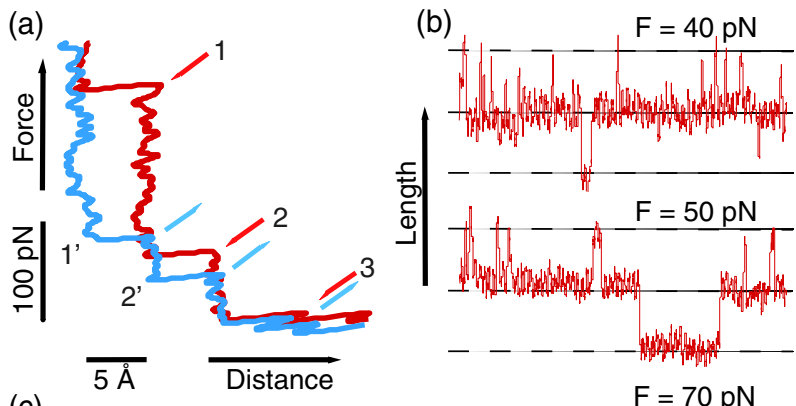

(c)
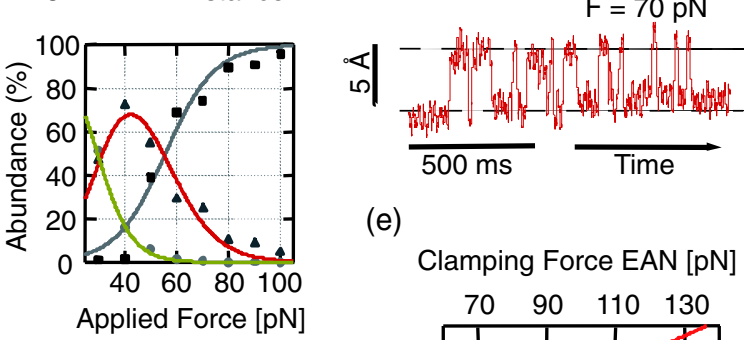

(e)
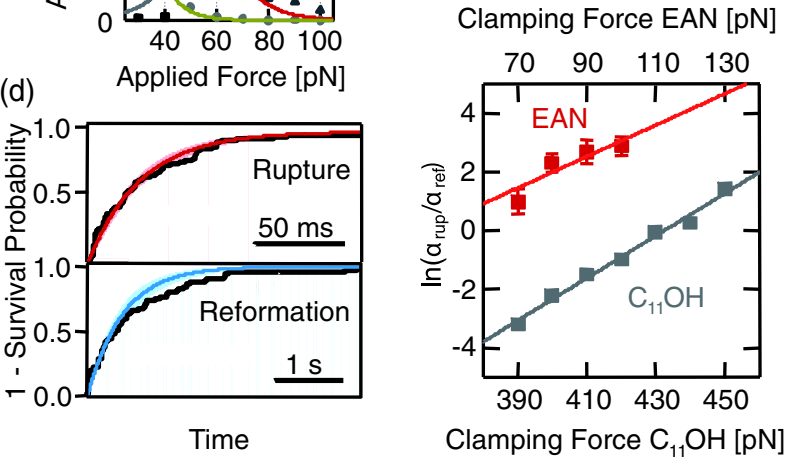

FIG. 3 (color online). The solvation dynamics of EAN. (a) Force extension trajectories showing the rupture and reformation of 3 confined EAN solvation layers. (b) The system hops between layers 1 and 2 as the applied constant force varies from $70-40 \mathrm{pN}$. (c) Relative abundances of the reformed (green) and ruptured state (red) of layer 1 and the ruptured state of layer 2 as a function of the applied force. Global fit of Eq. (2) yields $\Delta l=4.3 \pm 0.4 \AA$ and $\Delta G=(5.9 \pm 0.6) k T$ (layer 2, red line); $\Delta l=6.2 \pm 0.1 \AA$ and $\Delta G=(4.5 \pm 1) k T$ (layer 3, gray line). (d) The cumulative density function reconstructed from the transition dwell times is fitted, as a first approximation, to an exponential function to obtain the rates of rupture (red line, $\tau=29 \pm 4 \mathrm{~ms}$ ) and reformation (blue line, $\tau=500 \pm 100 \mathrm{~ms}$ ) for $F=100 \mathrm{pN}$. (e) Fitting of Eq. (4) yields $\Delta G=(4.5 \pm 1) k T$ and $N=2.0 \pm$ 0.8 (EAN, red solid line). For comparison, the same equation was fitted to 1-undecanol results (gray solid line) yielding $\Delta G=(31 \pm 2) k T$ and $N=0.9 \pm 0.1$. 
SFA [24] and AFM results $[9,25]$. This suggests that the $\mathrm{EA}^{+}$and the nitrate ions facing the bulk liquid are perpendicularly adsorbed onto the mica substrate. Because of the similar forces between consecutive solvation layers in EAN [Fig. 3(a)], applying a constant pushing force of $50 \mathrm{pN}$ to the EAN-mica interface results in an equilibrium situation where the system hops between three well-defined states (layers 1-2) in discrete jumps of $\sim 5 \pm 2 \AA$ [Fig. 3(b)]. Slightly changing the applied force to lower $(40 \mathrm{pN})$ or higher $(70 \mathrm{pN})$ values dramatically shifts the equilibrium properties of the system. The relative time abundance of each state as a function of the applied force is shown in Fig. 3(c). Fitting Eq. (2) for each state provides a direct measurement of the associated $\Delta G$ and $\Delta l$ values for the studied layers 1 and 2. Crucially, the obtained $\Delta l$ values are $\sim 5 \AA$, thus corresponding to the actual measurement obtained from force-extension [Fig. 3(a) and Fig. S9 in [18]] and forceclamp (Fig. S10, [18]). As before, the dwell times for each individual transition can be used to compute the cumulative probability density function for the rupturing and reformation processes, the time course of which can be in both cases captured by a single exponential [Fig. 3(d)]. The resulting rates at each particular force are fitted to Eq. (4) yielding in this case $N=2.0 \pm 0.8 \approx 2$ [Fig. 3(e)]. The observed $N=2$ indicates that 2 interactions are disrupted in parallel, most likely suggesting that the ions have to be dislocated in pairs in order to avoid configurations where two ions of the same sign are in close contact.

While qualitatively similar, AFM solvation force measurements quantitatively differ from SFA results [23]. The main important difference lies in the massively different ( $\sim 10^{6}$ fold) contact radius, which affects the liquid confinement area and hence the mechanisms of liquid squeeze-out. Briefly, the nanometer confinement below the AFM tip has allowed measurement of solvation forces in branched liquid molecules lacking molecular symmetry [6], and the packing properties can be analysed even for surfaces that are not atomically flat (such as self-assembled monolayers, SAMs) [23], both being important prerequisites in SFA measurements [1]. Moreover, the large temperature effect of the solvation forces observed with AFM [26] and the lack of correlation between the measured forces and the tip radius [27] - indicating that the nanometer-scale microasperities of the tip dominate the short-range interaction-suggest novel molecular mechanisms underlying the squeezing out of liquids confined in the gap between the cantilever tip and the substrate [23]. These observations have been recently complemented by AFM and STM imaging [16,28,29], directly demonstrating the semisolid behavior of the liquid layers. Our results using force-clamp spectroscopy add a new dimension to the study of the solvation phenomena, providing precise quantification of the equilibrium rupturereformation dynamics, which allow, for the first time, the reconstruction of the solvation free-energy landscape. Moreover, these experiments provide new insights into
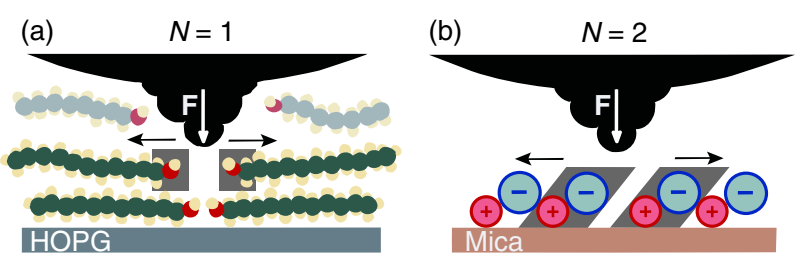

FIG. 4 (color online). Schematics of the rupturing process for (a) 1-undecanol $(N=1)$ and (b) EAN $(N=2)$.

the (molecular) mechanisms underlying liquid disruption; (i) using the Bell formalism, borrowed from antigenantibody and single molecule mechanics pulling experiments, we gained access to the number of key interactions disrupted (and reformed) under force. (ii) For 1-undecanol, molecules lie parallel to the HOPG surface. Our experiments reveal that a unique interaction $(N=1)$ needs to be broken to induce the rupture of the layer. We speculate that the pore created in the structure of the confined liquid induces the lateral shift of a whole row of molecules pushed to the sides [Fig. 4(a)]. However, our experiments cannot tell whether the lateral displacement corresponds to 1 or more molecular positions. (iii) In the case of EAN, molecules are oriented upright from the mica normal direction. The $\mathrm{EA}^{+}$moiety measures $3 \AA$, while the nitrate measures $1.7 \AA$ [30]. Since the barrier is highly symmetric $(\Delta x=2.5 \AA)$, and probably located in the plane where electrostatic interactions take place, we speculate that there is a slight molecular mismatch between the position of contiguous ion pairs in order to better accommodate their charges. Our experiments show that in this case $N=2$. Since $N$ only corresponds to the number of molecules or interactions being disrupted in parallel with the tip, we hypothesise that individual anion-cation pairs need to be displaced together in tandem [Fig. 4(b)], which is easy to rationalize due to their electrostatic nature. These results demonstrate the surprisingly short-range dependence of the interaction between the last atom of the AFM probe and the solvation layer. Altogether, our experiments directly capture the molecular motions involved in the rupturing and self-assembly processes of individual solidlike liquid layers and highlight the single molecule nature of the squeeze-out mechanism when the confinement area lies in the nanometer realm.

We thank Amy Beedle and Aisling Williams for critical reading of the manuscript. J. R.-G. was recipient of a "La Caixa" fellowship. This work was supported by the Marie Curie CIG (293462), BBSRC (J00992X/1), and Royal Society (RG120038) Grants, and by the EPSRC Fellowship (K00641X/1), all to S. G.-M.

* Corresponding author. sergi.garcia-manyes@kcl.ac.uk

[1] J. N. Israelachvili, 3rd ed. Intermolecular and Surface Forces (Academic Press, Elsevier, New York, 2011). 
[2] R. G. Horn and J. N. Israelachvili, J. Chem. Phys. 75, 1400 (1981).

[3] B. Cappella and G. Dietler, Surf. Sci. Rep. 34, 1 (1999).

[4] H. H. Butt and R. Stark, Colloids Surf. A 252, 165 (2005).

[5] F. Liu, S. de Beer, D. van den Ende, and F. Mugele, Phys. Rev. E 87, 062406 (2013).

[6] R. Lim and S. J. O'Shea, Phys. Rev. Lett. 88, 246101 (2002).

[7] N. N. Gosvami, S. K. Sinha, and S. J. O’Shea, Phys. Rev. Lett. 100, 076101 (2008).

[8] V. Franz and H. J. Butt, J. Phys. Chem. B 106, 1703 (2002).

[9] R. Atkin and G. G. Warr, J. Phys. Chem. C 111, 5162 (2007).

[10] R. Hayes, G. G. Warr, and R. Atkin, Phys. Chem. Chem. Phys. 12, 1709 (2010).

[11] J. I. Kilpatrick, S. H. Loh, and S. P. Jarvis, J. Am. Chem. Soc. 135, 2628 (2013).

[12] M. Antognozzi, A. D. L. Humphris, and M. J. Miles, Appl. Phys. Lett. 78, 300 (2001).

[13] M. Schlierf, H. B. Li, and J. M. Fernandez, Proc. Natl. Acad. Sci. U.S.A. 101, 7299 (2004).

[14] F. Mugele, S. Baldelli, G. A. Somorjai, and M. Salmeron, J. Phys. Chem. B 104, 3140 (2000).

[15] K. Morishige and T. Kato, J. Chem. Phys. 111, 7095 (1999).

[16] T. Hiasa, K. Kimura, and H. Onishi, J. Phys. Chem. C 117, 5730 (2013).

[17] L. Y. Low, J. Am. Stat. Assoc. 78, 987 (1983).
[18] See Supplemental Material at http://link.aps.org/ supplemental/10.1103/PhysRevLett.114.258303 for a full description of the materials and methods employed, and plots displaying the results of the supporting experiments.

[19] G. I. Bell, Science 200, 618 (1978).

[20] C. Cecconi, E. A. Shank, C. Bustamante, and S. Marqusee, Science 309, 2057 (2005).

[21] S. Garcia-Manyes, L. Dougan, C. L. Badilla, J. Brujic, and J. M. Fernandez, Proc. Natl. Acad. Sci. U.S.A. 106, 10534 (2009).

[22] I. Popa, J. M. Fernandez, and S. Garcia-Manyes, J. Biol. Chem. 286, 31072 (2011).

[23] S. J. O'shea, N. N. Gosvami, L. T. W. Lim, and W. Hofbauer, Jpn. J. Appl. Phys. 49, 08LA01 (2010).

[24] R. G. Horn, D. F. Evans, and B. W. Ninham, J. Phys. Chem. 92, 3531 (1988).

[25] D. Wakeham, R. Hayes, G. G. Warr, and R. Atkin, J. Phys. Chem. B 113, 5961 (2009).

[26] L. T. Lim, A. T. Wee, and S. J. O'Shea, J. Chem. Phys. 130, 134703 (2009).

[27] S. J. O'shea and M. E. Welland, Langmuir 14, 4186 (1998).

[28] W. Hofbauer, R. J. Ho, R. Hairulnizam, N. N. Gosvami, and S. J. O'Shea, Phys. Rev. B 80, 134104 (2009).

[29] T. Hiasa, K. Kimura, and H. Onishi, J. Phys. Chem. C 116, 26475 (2012).

[30] Y. Umebayashi, W.-L. Chung, T. Mitsugi, S. Fukuda, M. Takeuchi, K. Fujii, T. Takamuku, R. Kanzaki, and S. Ishiguro, J. Comput. Chem., Jpn. 7, 125 (2008). 